\title{
Correction to: Nonthermal Plasma Technology
}

\author{
Ximena V. Yepez, N. N. Misra, and Kevin M. Keener
}

\section{Correction to:}

Chapter 23 in A. Demirci et al. (eds.), Food Safety

Engineering, Food Engineering Series, https://doi.org/10.1007/978-3-030-42660-6

In the original version of this book, the country name in the affiliation of author Kevin M. Keener was printed incorrectly as Canada, which should be USA. This has been updated in this revised version of the book. 